\title{
Dual-Modality Probe for Characterization of Heterogeneous Mixtures
}

\author{
T. Dyakowski, J. M. Hale, A. Jaworski, N. M. White, Senior Member, IEEE, A. Nowakowski, G. Meng, and S. Rwifa
}

\begin{abstract}
This paper presents a laboratory feasibility study aiming at the development of a dual modality sensor for development within an oil and gas extraction plant to measure the composition of heterogeneous mixtures. The technology of manufacturing the sensor is briefly described and the preliminary measurements, conducted for mixtures of vegetable oil and saline water, using two modalities: ultrasound and electrical, are presented. The experimental results obtained from ultrasonic measurements are compared with theoretical predictions. Finally, conclusions and recommendations for future work are presented.
\end{abstract}

Index Terms-Heterogeneous mixtures, speed of sound, thickfilm, ultrasonic.

\section{INTRODUCTION}

$\mathbf{T}$ HE AIM OF THE presented study is to develop a reliable dual modality sensing technique to be deployed in the hostile environment of an oil and gas extraction plant, particularly within primary separation systems (both top-side and subsea) or, potentially, within the down-hole environment. Here, the heterogeneous mixtures consist of at least four components: crude oil, formation water, gas, and solid particulates. Typical operating conditions of such installations are characterized by high temperature reaching in excess of $150{ }^{\circ} \mathrm{C}$ pressure in excess of $150 \mathrm{bar}$, and the presence of highly aggressive chemical compounds. From the above, it is clear that any possible sensor design must meet at least two criteria: to survive the high operating pressure and temperatures and to ensure the chemical stability of the components exposed to process media.

Generally, heterogeneous mixtures of multiple phases or components are a common occurrence within many industrial processes; numerous examples can be given from areas as diverse as chemical, petrochemical, oil and gas extraction, pharmaceutical, food and drink and water treatment plants. The

Manuscript received January 16, 2004; revised July 23, 2004. The associate editor coordinating the review of this paper and approving it for publication was Dr. Krikor Ozanyan.

T. Dyakowski is with the Department of Chemical Engineering, University of Manchester Institute of Science and Technology, Manchester M60 1QD, U.K., and also with the Computer Engineering Department, Technical University of Lodz, Lodz, Poland (e-mail: tom.dyakowski@umist.ac.uk).

J. M. Hale is with the School of Mechanical and Systems Engineering, University of Newcastle upon Tyne, Newcastle NE1 7RU, U.K. (e-mail: jack.hale@newcastle.ac.uk).

A. Jaworski and G. Meng are with the School of Engineering, University of Manchester, Manchester M13 9PL, U.K. (e-mail: a.jaworski@man.ac.uk).

N. M. White is with the School of Electronics and Computer Science, University of Southampton, Southampton SO17 1 BJ, U.K. (e-mail: nmw@ecs.soton.ac.uk).

A. Nowakowski and S. Rwifa are with the Department of Chemical Engineering, University of Manchester Institute of Science and Technology, Manchester, Manchester M60 1QD, U.K.

Digital Object Identifier 10.1109/JSEN.2005.843902 ability to monitor and control the multiphase phenomena is therefore one of the most fundamental issues.

\section{Probe Design and Manufacture}

A dual modality probe using two measurements modalities: electrical and ultrasound, was designed, manufactured, and tested in laboratory conditions. The probe is built from two identical transducers. Each of them consists of two electrodes with a piezoelectric layer connected to the first and second electrode. The first electrode, second electrode, and piezoelectric layer of each transducer form a pressure wave transducer. Both transducers are displaced from one another such that the second electrodes from both transducers can be used for electrical measurements.

The fabrication of the transducers was undertaken at the University of Southampton within the School of Electronics and Computer Science. The devices were made using thick-film technology, with which the Southampton group have considerable experience, [1]. The sensors comprise three layers of thick-film material: a lower electrode layer, a piezoelectric layer, and an upper electrode layer. The substrate material was $96 \%$ alumina of thickness $635 \mu \mathrm{m}$. Each electrode layer was fabricated with a commercial gold paste and the piezoelectric layer was a special-purpose paste developed at the University. Lead zirconate titanate (PZT) powder type 5A, manufactured by Morgan Electronics, Ltd., was chosen as the active material for the piezoelectric paste. This has a Curie temperature exceeding $170^{\circ} \mathrm{C}$, allowing the sensors to operate at the desired specification of $150{ }^{\circ} \mathrm{C}$, as fully discussed elsewhere.

The size of the lower gold electrode is $10 \times 10 \mathrm{~mm}$ and this is screen-printed onto the alumina substrate, dried in an infrared drier at about $1500^{\circ} \mathrm{C}$ and then fired in a belt furnace at a peak temperature of $8900{ }^{\circ} \mathrm{C}$. The thick-film PZT layer was printed twice in order to obtain a thickness of around $50 \mu \mathrm{m}$ and then subjected to a similar firing profile to the initial layer. The upper gold layer, of dimensions $25 \times 25 \mathrm{~mm}$, was then printed, dried, and fired in the same manner to the other layers. The resulting device is essentially a planar capacitor-type structure. After processing, the PZT layer has to be polarized in order to induce piezoelectric behavior. This is achieved by placing the substrate on a hot plate at a temperature of around $890^{\circ} \mathrm{C}$ and applying a dc electric field of $4 \mathrm{MV} / \mathrm{m}$ across the sample for $10 \mathrm{~min}$.

\section{EXPERIMENTAL SETUP}

Pairs of transducers, as shown in Fig. 1, were attached to a fork-like probe, as shown in Fig. 2 in such a way that the measurement of the time of flight would conduct along a $25-\mathrm{mm}$ 




Fig. 1. Layout of the thick-film ultrasound sensor. a)

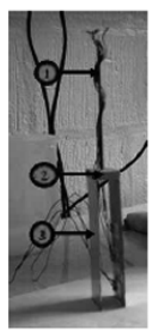

b)

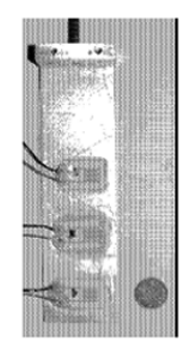

Fig. 2. (a) Fork-like probe used for supporting transducers. 1: support rod; 2: base plate; 3: aluminum "leaves" supporting the transducers. (b) An array of transducers on one of the aluminum "leaves."

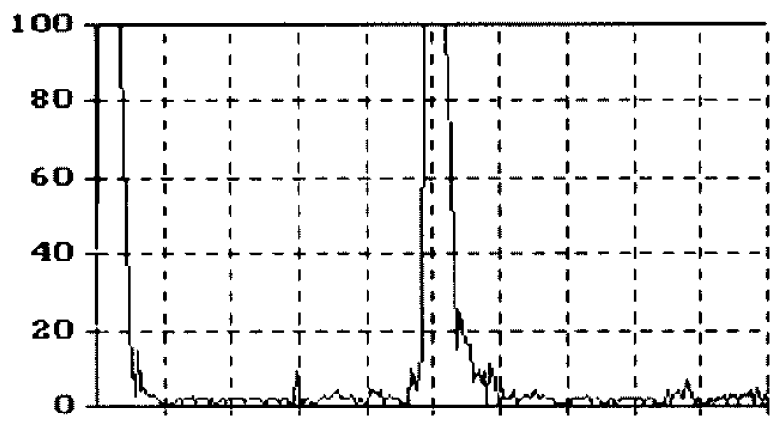

Fig. 3. Typical waveform of the sent and received signal obtained during experiments (arbitrary scale).

path in the horizontal direction between the transducers immersed in the mixture. The transducers were insulated with an epoxy layer with the thickness of $0.2 \mathrm{~mm}$.

An ultrasonic flaw detector, EPOCH II, Model 2100, was used to excite one of the transducers while the other transducer acted as a receiver. The waveform of the excitation and the received signal were displayed on a computer screen connected to the flaw detector, which allowed the measurement of the time of flight of the ultrasonic wave between the two transducers.

Fig. 3 shows a typical waveform graph obtained in the experiments. From the waveform of the detector, the time between the excitation peak (the first peak in the waveform) and the receiving peak (the second peak in the waveform) can be read. With the distance between the two transducers $(25 \mathrm{~mm})$, and taking into account the time of flight within the layers of transducers insulation, the ultrasonic velocity can be determined. The trend of the speed of sound with the change of temperature was also investigated.

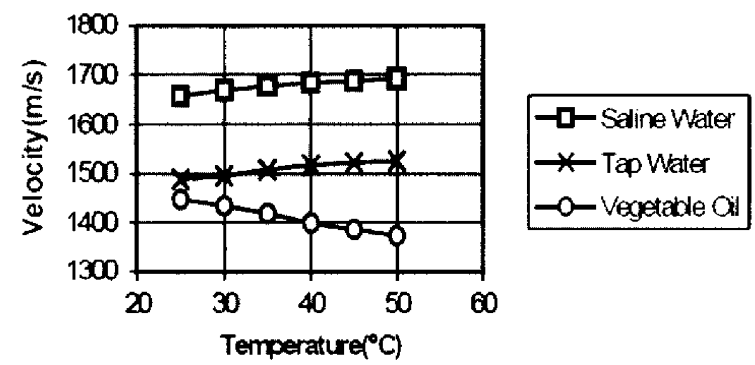

Fig. 4. Speed of sound in salty water and oil at different temperatures (data for tap water for reference).



Fig. 5. Speed of sound in emulsions at different oil concentration and at different temperatures.

At the initial stage of the investigation, fluids present in the oil and gas extraction were simulated by the use of vegetable oil and saline water with a sodium chloride content of $20 \%$ by weight. They were used for both the safety aspects of the laboratory and the convenience of using relatively clean fluids. The high content of sodium chloride in saline water was chosen to mimic the density of the formation water present in oil extraction processes.

In order to obtain stable mixtures (emulsions), an aqueous surfactant solution was prepared by dissolving $1 \%$ by weight of Tween 20 (ICI Chemical \& Plymers, Ltd.) in saline water. A series of vegetable oil-in saline water emulsions with volume concentration of oil varying between $10 \%$ and $50 \%$ in steps of $10 \%$ were prepared by blending the components in a glass beaker with a homogenizer (IKA-EUROTURRAX T20) at a rotational speed of $22000 \mathrm{rpm}$. Similarly, a series of saline water in-oil emulsions with volume concentrations of saline water between $10 \%$ and $50 \%$ in steps of $10 \%$ were prepared using a similar procedure. In the latter case, to avoid confusion, instead if measuring water contents in oil, the mixtures are identified by volume fraction which varies between $59 \%$ and $100 \%$. The Distribution Modal Size of droplets in the emulsions tested were in the range of 1.0-10.0 $\mu \mathrm{m}$, as fully described elsewhere [2].

\section{EXPERIMENTAL RESULTS}

\section{A. Ultrasonic Measurements}

1) Speed of sound in pure liquids Initial measurements were done for pure liquid and the speed of sound at different temperature was investigated. The results shown in Fig. 4 indicate that the speed of sound in saline water increases with the increase of temperature while the speed in vegetable oil decreases with the increase of temperature. 
a)

\section{Capacitance (C) vs. oil percentage}

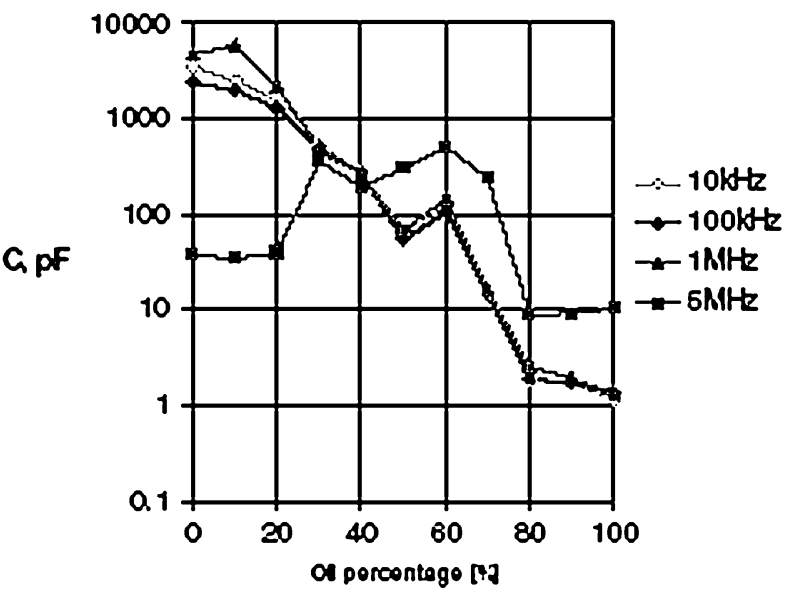

b)

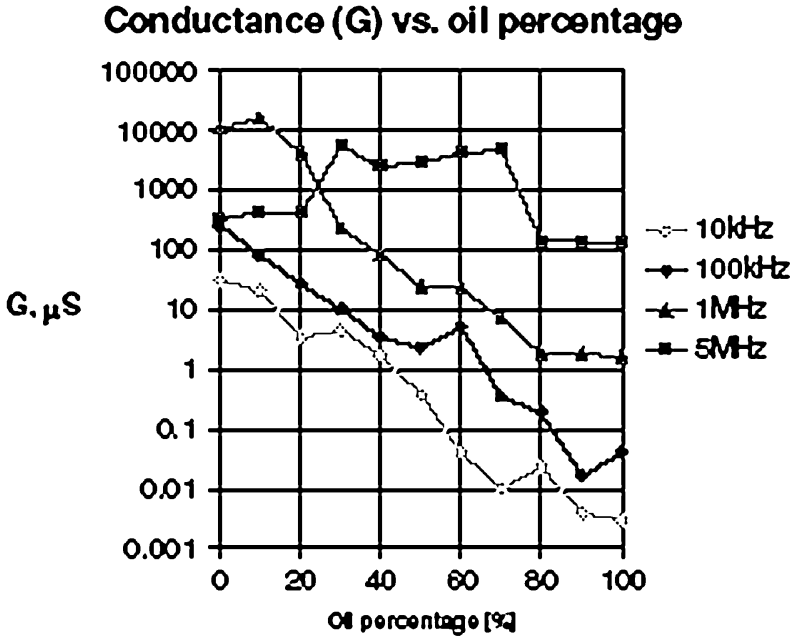

Fig. 6. Impedance measurements obtained using ac circuit: (a) capacitance and (b) conductance.

2) Speed of sound in heterogeneous mixtures

The second part of the investigation involved measurement of the sound velocity in vegetable oil and saline water emulsions. The measured velocity of sound versus oil volume fraction at different temperatures is shown in Fig. 5.

\section{B. Electrical Measurements}

The effect of varying frequency of electrical field on the measured capacitances and conductances is depicted in Fig. 6. The results of measuring the capacitance values using a charge-discharge circuit are presented elsewhere, [3]. The results shown in Fig. 6 were obtained using an impedance analyzer. Except a drastic change in the results for a frequency of $5 \mathrm{MHz}$, the measured capacitances are nearly independent of the applied frequencies, as illustrated in Fig. 6(a), whereas the conductance data shows a clear dependence on the applied frequency, as shown in Fig. 6(b).

\section{Analysis of Ultrasound Data}

A model describing the ultrasonic propagation in heterogeneous mixtures was $\gamma$ developed by Yi et al. [4] and Tsouris and Tavlarides [5]. It predicts the time of flight of the ultrasonic signal as a function of the volume fraction of the dispersed phase by taking into account the change in the length of the sound path arising from reflection and refraction effects at the droplet interface.

Equation (1) summarizes the model obtained

$$
\Phi=\left(t^{*}-t_{c}\right) /\left(g_{d} t_{d}-g_{c} t_{c}\right)
$$

where $\Phi$ is the volume fraction, $t^{*}$ - time of flight in dispersion, $t_{c}$ - time of flight in the continuous phase; and $t_{d}$ - time of flight in the dispersed phase.
The correction factors $g_{d}, g_{c}$ for each phase account for the sound refraction phenomena and are given by the following relations:

$$
\begin{aligned}
\mathrm{g}_{\mathrm{d}}= & 1 / \gamma\left[1-\left(1-\gamma^{2}\right)^{3 / 2}\right] \\
\mathrm{g}_{\mathrm{c}}= & 1+1 \gamma^{3} /\left[1-\left(1-\gamma^{2}\right)^{3 / 2}\right] \\
& -3 / 5\left[1-\left(1-\gamma^{2}\right)^{5 / 2}\right]-2 / 5 \gamma^{2}
\end{aligned}
$$

where $\gamma$ is the ratio of the sound velocity in the dispersed phase to the sound velocity in the continuous phase. Equations (2) and (3) hold for $\gamma \leq 1$ which implies that the dispersed phase needs to have a lower sound speed than the continuous phase. This will be important for the results presented.

The relationship between speed of sound in the mixture and the volume fraction can be achieved by reorganizing (1)

$$
1 / \mathrm{v}^{*}=\Phi\left(\mathrm{g}_{\mathrm{d}} / \mathrm{v}_{\mathrm{d}}-\mathrm{g}_{\mathrm{c}} / \mathrm{v}_{\mathrm{c}}\right)+1 / \mathrm{v}_{\mathrm{c}}
$$

where $\mathrm{v}^{*}$ is the speed of sound in the dispersion, $\mathrm{v}_{\mathrm{c}}$ is the speed of sound in the continuous phase, and $\mathrm{v}_{\mathrm{d}}$ is the speed of sound in the dispersed phase. The sound velocity at a given temperature in a pure phase is constant, so there is a linear relationship between the volume fraction and the reciprocal of ultrasonic velocity in the mixed states.

The comparison of the experimental results with theoretical model is presented here for the temperature of $25^{\circ} \mathrm{C}$. Similar results can be obtained for other temperatures. From the measurements conducted in pure liquids, one can obtain the value of $\gamma$ in (2) and (3). At $25^{\circ} \mathrm{C}$ for the speed of sound in vegetable oil of $1446.8 \mathrm{~ms}^{-1}$ and that in saline water of $1700.6 \mathrm{~ms}^{-1}$, the value of $\gamma$ is 0.85 . Equation (4) will then assume the following form:

$$
1000 / \mathrm{v}^{*}=0.0013 \Phi+0.5880 .
$$

It is clear from (4) and (5) that there is a linear relationship between the reciprocal of the speed of sound and the volume fraction. Therefore, it is more convenient to present data from 




Fig. 7. Trend of $1000 / \mathrm{V}$ versus oil percentage at $25^{\circ} \mathrm{C}$.

Fig. 5 in the form shown in Fig. 7, where on the vertical axis the inverse of velocity multiplied by a factor of 1000 is plotted.

The data in Fig. 7 can be subjected to linear regression process. Given the condition that $\gamma \leq 1$, this can only be done for the first six points on the left of Fig. 7. The relationship obtained is given as (6)

$$
1000 / \mathrm{v}^{*}=0.0012 \Phi+0 .
$$

It shows that the regression curve coincides almost identically with the theoretical prediction. Therefore, the theory explained with (1)-(5) can be applied to predict the fraction of the oil-insaline water mixtures.

For oil volume fractions between $50 \%$ to $100 \%$, there is no mathematical model $(\gamma>1)$ available. However, it may be interesting to note that the regression analysis for this range yields the following relationship:

$$
1000 \mathrm{v}^{*}=0.008 \Phi+0.6069 .
$$

Referring to Fig. 7, it can be seen that sound velocity in the emulsion investigated decrease with increase in the oil fraction in emulsions. From Fig. 4, the results show that the speed of sound in oil-saline water emulsions decreases with the increases of temperatures.

\section{CONCLUSION}

The concept of designing a dual-modality probe by using a thick-film technology, for metering the oil/water composition, was validated in laboratory conditions. The designed transducer consist of a layer sandwich structure. It is hoped that the materials used for the sensors will allow the sensor to survive high operating temperatures and pressures as well as contact aggressive media.

The probe has shown promising measurement characteristics in both ultrasonic and electrical modalities. Ultrasonic characteristics have been verified against a simple theoretical model.

Future work needs to concentrate on the following:

- long-term testing of the sensors in harsh conditions and evaluation of their performance;

- studies of the electrical modality in order to explain the character of the measurement obtained;

- application of dielectric coating on the sensor, most likely in the form of layer of glass sintered on the surface, and characterization of such a modified sensor design.

\section{REFERENCES}

[1] N. M. White and D. Turner, "Thick-film sensors: Past, present and future," Meas. Sci. Technol., vol. 8, pp. 1-20, 1997.

[2] T. Dyakowski, J. Hale, A. Jaworski, N. White, N. Harris, A. Nowakowski, G. Meng, and S. Rwifa, "Characterization of heterogeneous mixtures by using thick-film ultrasonic transducers," in Proc. 3rd World Congr. Industrial Process Tomography, Banff, ON, Canada, Sept., 2-5 2003.

[3] A. Jaworski, T. Dyakowski, and G. A. Davies, "A capacitance probe for interface detection in oil and gas extraction plant," Meas. Sci. Technol., vol. 10, pp. L15-L20, 1999.

[4] C. Tsouris and L. Tavlarides, "Comments on model for hold-up measurements in liquid dispersions using an ultrasonic technique," IEC Res., vol. 29, pp. 2170-2172, 1990

[5] C. Tsouris and L. Tavlarides, "Control of dispersed-phase volume fraction in multistage extraction columns," Chem. Eng. Sci., vol. 46, no. 11, pp. 2857-28656, 1991.

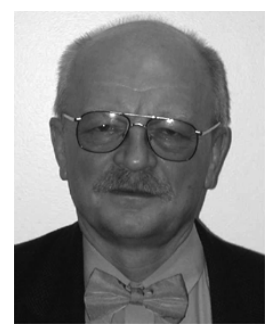

T. Dyakowski is a Professor with the Department of Chemical Engineering, University of Manchester Institute of Science and Technology (UMIST), Manchester, U.K., and Manager of the Applied Process Tomography Unit, UMIST. He has participated in and led research teams in a number of areas, including pneumatic conveying systems, solid-liquid separators, tomographic techniques for imaging multiphase flows, heat and mass transfer exchangers for refrigerators and heat pumps, and measurement techniques for monitoring oil-water separation processes. He has published over 120 research papers in these areas and has contributed to several books in addition to his own major research monographs. During the last ten years, he has been actively involved in developing instrumentation for multiphase flow monitoring and measurement-in particular, tomographic methods. He is recognized as an expert in this field and as a coauthor of major textbooks. The results of his research obtained for gas-solid systems within pneumatic lines and solid-liquid systems in hydrocyclones, fluidized beds, and fluidized risers have been extensively published. His most recent work was in the area of electrical tomography for the online and in situ monitoring of multiphase flow structures within process equipment.



J. M. Hale is the Reader in Structural Dynamics at the University of Newcastle upon Tyne, Newcastle, U.K. His research interests include structural monitoring and the development of dynamic measurement systems for use in hostile environments.

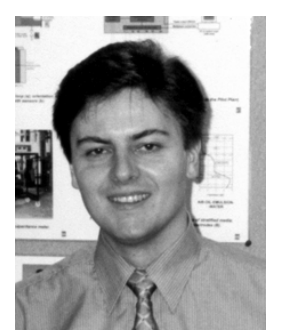

A. Jaworski received the degree in applied mechanics from the Warsaw University of Technology, Warsaw, Poland, in 1991, and the Ph.D. degree in the area of experimental fluid mechanics, which concerned a fundamental study of the vortex breakdown phenomenon, from Imperial College, London, London, U.K.

$\mathrm{He}$ is a Senior Lecturer and EPSRC Advanced Research Fellow (2004 to 2009) with the School of Mechanical, Aerospace, and Civil Engineering, University of Manchester, Manchester, U.K. His postdoctoral work in chemical engineering at the University of Manchester concerned experimental studies of multicomponent flows, the design of instrumentation for flow imaging in harsh industrial environments, and industrial process tomography. His recent interests relate to thermoacoustic technologies and the fundamentals of flow separation and boundary layer transition and their actuation using MEMS. 


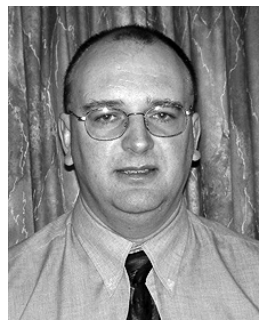

N. M. White (SM'02) received the Ph.D. degree with a thesis on the application of thick-film piezoresistors for load cells in 1988.

$\mathrm{He}$ is a currently a Professor of intelligent sensor systems within the School of Electronics and Computer Science, University of Southampton, Southampton, U.K., where he is also Director of the Institute of Transducer Technology. He was appointed Lecturer in 1990, Senior Lecturer in 1999, Reader in 2000 , and currently holds a Personal Chair. He has published extensively in the area of thick-film sensors and intelligent instrumentation and is the author or coauthor of over 100 scientific publications.

He is a Fellow of the Institute of Physics and the Institution of Electrical Engineers, a Chartered Engineer, and has served on several committees in various professional bodies.

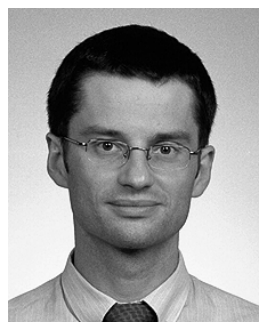

A. Nowakowski is a Lecturer of mechanical engineering at the University of Sheffield, Sheffield, U.K. His academic background is in applied and computational mechanics and his scientific interests are in the field of computational fluid mechanics. His research activity has been concentrated in the general area of aerodynamics and multicomponent and multiphase flows. In all of these categories, he has developed the algorithms to simulate relevant flow phenomena. His numerical applications have been used to solve the problems of practical interest, including the flow in hydrocyclones or for predicting the optimal configuration of newly designed flow meters.

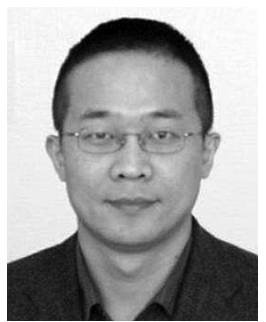

G. Meng received the M.S. degree in the area of thermal-fluid sciences from the Department of Thermal Engineering, Tongji University, China. He is currently pursuing the Ph.D. degree at the School of Engineering (currently the School of Mechanical, Aerospace, and Civil Engineering), University of Manchester, Manchester, U.K.

He has been working in the areas of industrial sensor design and on the development of measurement techniques, particularly electrical and ultrasonic methods.



S. Rwifa received the B.Sc., M.Sc., and Ph.D. degrees.

He has many years of experience working in chemical plants. This very valuable industrial experience makes him very enthusiastic about the understanding of the process tomography methodology. 